\title{
Equipe de enfermagem frente aos cuidados paliativos em pediatria: Revisão
}

\section{integrativa}

\author{
Nursing team facing palliative care in pediatrics: Integrative review \\ Equipo de enfermería frente a los cuidados paliativos en pediatría: Revisión integrativa
}

Recebido: 09/04/2021 | Revisado: 15/04/2021 | Aceito: 16/04/2021 | Publicado: 29/04/2021

\author{
Aline Paula Spibida Silvestri \\ ORCID: https://orcid.org/0000-0002-1424-2153 \\ Universidade de Passo Fundo, Brasil \\ E-mail: 166174@upf.br \\ Edilson Lima dos Santos \\ ORCID: https://orcid.org/0000-0002-1226-0665 \\ Prefeitura de Passo Fundo, Brasil \\ E-mail: edilson-san@hotmail.com \\ Gabriela Fagan Belini \\ ORCID: https://orcid.org/0000-0003-4492-2392 \\ Prefeitura de Passo Fundo, Brasil \\ E-mail: gabrielafagan @ hotmail.com \\ André Olivo Pellizzaro \\ ORCID: https://orcid.org/0000-0001-6461-1183 \\ Hospital de Clínicas de Passo Fundo, Brasil \\ E-mail: andreopellizzaro@gmail.com \\ Tamires da Silva Moura \\ ORCID: https://orcid.org/0000-0002-5606-5853 \\ Universidade de Passo Fundo, Brasil \\ E-mail: tamiressilvamoura@hotmail.com \\ Suelen Gonçalves \\ ORCID: https://orcid.org/0000-0001-5716-192X \\ Universidade de Passo Fundo, Brasil \\ E-mail:163876@upf.br \\ Marciele Begnini \\ ORCID: https://orcid.org/0000-0003-3893-8574 \\ Universidade de Passo Fundo, Brasil \\ E-mail: 173543@upf.br \\ Daniela Ramos Oliveira \\ ORCID: https://orcid.org/0000-0003-3880-041X \\ Universidade de Passo Fundo, Brasil \\ E-mail: danielaramos@upf.br \\ Angélica Moi \\ ORCID: https://orcid.org/0000-0002-3658-7224 \\ Wizard Idiomas de Serafina Corrêa, Brasil \\ E-mail: angelicapedagogico@gmail.com
}

\begin{abstract}
Resumo
Os cuidados paliativos são medidas de cuidados adotadas a partir do momento em que não existe mais a possibilidade de cura para o paciente. A enfermagem possui um papel fundamental nesse processo, oferecendo conforto e qualidade de vida a criança. $\mathrm{O}$ objetivo deste estudo foi compreender a abordagem paliativa da equipe de enfermagem em pediatria. Trata-se de uma revisão bibliográfica do tipo integrativa, a partir de publicações científicas indexadas nas bases de dados Literatura Latino-Americana e do Caribe em Ciências da Saúde (LILACS), Base de dados de Enfermagem (BDENF), MEDLINE, via Biblioteca Virtual em Saúde (BVS) e Scientific Electronic Library Online (SciELO), no período de 2015 a 2020. A análise dos dados foi realizada utilizando a temática de caracterização de dados, por meio desta, emergiram duas categorias: "Abordagens adotadas pela enfermagem em pacientes pediátricos em cuidados paliativos" e "Estratégias de enfrentamento da equipe diante das situações vivenciadas no processo de cuidados paliativos." Os estudos demonstram a importância do papel ocupado pela enfermagem neste processo, bem como, as dificuldades que são encontradas pela equipe e pelas famílias que vivenciam essas situações, por isso, além da assistência, a enfermagem é rede de apoio, de suporte ao paciente e seu familiar.
\end{abstract}

Palavras-chave: Cuidados paliativos; Enfermagem; Pediatria. 


\begin{abstract}
Palliative care is a measure of care adopted from the moment when the possibility of a cure for the patient no longer exists. Nursing has a fundamental role in this process, offering comfort and quality of life to the child. The aim of this study was to understand the palliative approach of the pediatric nursing team. This is an integrative bibliographic review, based on scientific publications indexed in the databases of Latin American \& Caribbean Health Sciences Literature (LILACS), Brazilian Nursing Database (BDENF), MEDLINE, via Virtual Health Library (VHL) and Scientific Electronic Library Online (SciELO), from 2015 to 2020. The analysis of the data was performed using the theme of data characterization, through which two categories emerged: "Approaches adopted by nursing in pediatric patients in palliative care" and "Team coping strategies in the face of situations experienced in the palliative care process". The studies show the importance of the role played by nursing in this process, as well as, the difficulties that are encountered by the team and by the families that experience these situations, hence, in addition to assistance, nursing is a support network, support for patients and their families.
\end{abstract}

Keywords: Palliative care; Nursing; Pediatrics.

\title{
Resumen
}

Los cuidados paliativos son medidas de cuidados adoptadas a partir del momento en que deja de existir la posibilidad de cura para el paciente. La enfermería tiene un papel fundamental en este proceso, ofreciendo comodidad y calidad de vida a los niños. El objetivo de este estudio fue comprender el abordaje paliativo del equipo de enfermería en pediatría. Se trata de una revisión bibliográfica integrativa., a partir de publicaciones científicas indexadas en las bases de datos Literatura Latinoamericana y del Caribe en Ciencias de la Salud (LILACS), Base de datos de enfermería (BDENF), MEDLINE, vía Biblioteca Virtual en Salud (BVS) y Scientific Electronic Library Online (SciELO), en el período de 2015 a 2020. El análisis de los datos fue realizado utilizando la temática de caracterización de datos, y por medio de esta, emergieron dos categorías: "Abordajes adoptados por la enfermería en pacientes pediátricos en cuidados paliativos" y "Estrategias de enfrentamiento del equipo ante situaciones vividas en el proceso de cuidados paliativos. Los estudios demuestran la importancia del papel ocupado por la enfermería en este proceso, así como, las dificultades encontradas por el equipo y por las familias que viven estas situaciones, por eso, además de la asistencia, la enfermería es una red de apoyo, de soporte al paciente y sus familiares.

Palabras clave: Cuidados paliativos; Enfermería; Pediatría.

\section{Introdução}

O cuidado paliativo é uma abordagem multiprofissional de assistência, aplicada a pacientes sem possibilidade de cura, de forma integrada e individualizada, adaptada ao tratamento, a fim de melhorar a qualidade de vida e controlar os sintomas da doença (INCA, 2018). Além disso, tem o intuito de amenizar a angústia frente às possíveis mudanças físicas e psicológicas e transmitir informações que possam manter um vínculo próximo entre paciente e equipe (Hermes \& Lamarca, 2013).

Os cuidados paliativos em pediatria abrangem diversos aspectos, tais como, o desenvolvimento de conhecimentos para manejar os muitos sintomas físicos, psíquicos e as manifestações do sofrimento familiar, social e espiritual decorrentes da doença (Melo, Valero \& Menezes, 2013). Esses cuidados são implementados e individualizados àquela criança, pois algumas enfermidades em pacientes pediátricos, vão além da dor de todos que vivenciam neste meio, sofrem emocionalmente, por isso, a partir desse momento, paciente, família e equipe de saúde enfrentam desafios na busca e implementação de condutas para a melhoria da qualidade de vida (Misko et al., 2015).

Crianças com doenças crônicas encontram-se em situação ainda mais delicada, pois é comum que pais e profissionais negligenciam sua crescente habilidade em participar de decisões (Carvalho, 2016). Entretanto, é essencial o acompanhamento desta evolução, levando em conta a maturidade e a percepção para conceder o adequado respeito à autodeterminação destes indivíduos (Iglesias, Zollner \& Constantino, 2016).

As patologias que podem levar o paciente à cuidados paliativos são condições para as quais seja a única e melhor forma de tratamento, ou seja, doenças progressivas como cardiopatias congênitas, imunodeficiências graves, doenças metabólicas progressivas e encefalopatia crônica, porém, a patologia que mais comumente leva aos cuidados paliativos é o câncer, que por vezes submete a criança às inúmeras abordagens, até que se encontre a melhor forma de proporcionar conforto durante as etapas da doença (Carvalho, 2009).

A equipe de enfermagem representa uma força de trabalho indispensável para a garantia da prestação de um cuidado 
seguro e eficaz aos pacientes (Cavalcante et al., 2015). Ela atua de modo interdisciplinar visando reduzir o sofrimento e promover conforto e dignidade humana ao paciente e seu familiar, demonstrando compromisso com os cuidados paliativos em prol de um cuidar que tem a qualidade de vida como o principal objetivo durante esse processo (Alves et al., 2015). Além de proporcionar uma melhor comodidade ao paciente, atuar como rede de apoio possibilita que os medos e ansiedades sejam expressos, deixandoos mais seguros e acolhidos. Frente a essa problemática, tem-se a seguinte pergunta de pesquisa: "Quais são as estratégias de cuidados adotadas pela enfermagem para criança em cuidados paliativos?”. O objetivo deste estudo, foi compreender a abordagem paliativa na pediatria pela equipe de enfermagem.

\section{Método}

Trata-se de uma revisão integrativa, realizada por meio de levantamento bibliográfico. A pesquisa bibliográfica é uma das melhores maneiras de iniciar um estudo, comparando as semelhanças e diferenças entre os artigos. A revisão integrativa abrange a inclusão de estudos experimentais e não-experimentais para análise do fenômeno estudado. Implica em um vasto leque de propósitos: definição de conceitos, revisão de teorias e evidências e análise de problemas metodológicos de modo particular (Souza, Silva \& Carvalho, 2010).

A revisão integrativa é baseada em seis etapas. A primeira delas é a identificação do tema e a seleção de hipóteses ou questões de pesquisa para após realizar a revisão, sendo a norteadora desse processo. A segunda etapa determina os critérios de inclusão e exclusão. E, na terceira, ocorre a definição das informações que são extraídas dos estudos selecionados, organizandoas de maneira concisa (Mendes, Silveira \& Galvão, 2008). Na quarta etapa, é realizada a análise minuciosa e crítica dos artigos. A quinta, é a análise e interpretação dos resultados, nela é realizada uma comparação do conhecimento teórico, a identificação de conclusões e implicações resultantes da revisão integrativa. Já, durante a sexta etapa, há a apresentação da revisão/síntese do conhecimento (Mendes, Silveira \& Galvão, 2008). A Figura 1 apresenta de forma sucinta essas etapas.

Figura 1 - Etapas da Revisão Integrativa.

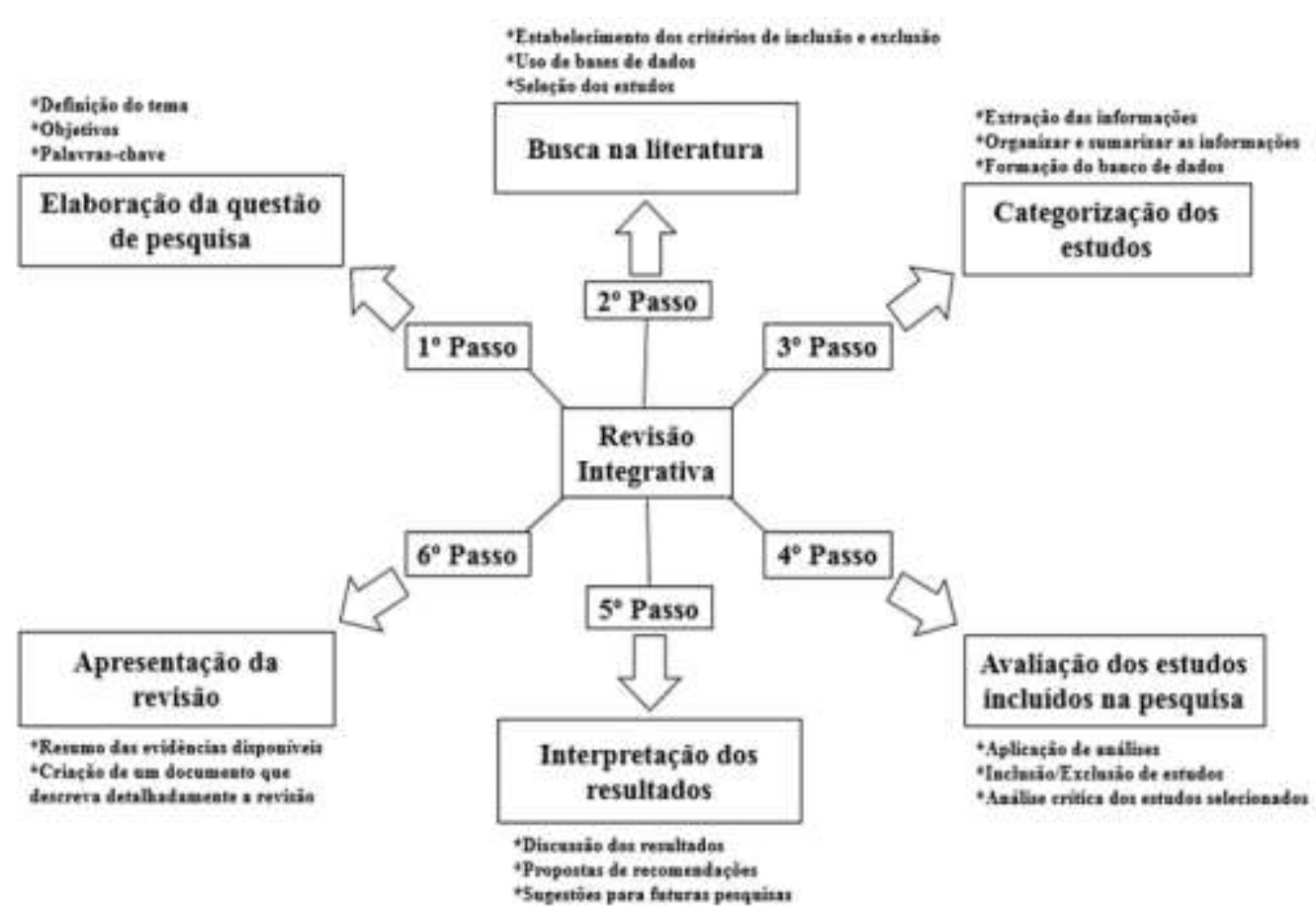

Fonte: Mendes, Silveira e Galvão, (2008). 
Para o levantamento dos artigos na literatura, realizou-se uma pesquisa nas seguintes bases de dados: Literatura LatinoAmericana e do Caribe em Ciências da Saúde (LILACS), BDENF e MEDLINE, via Biblioteca Virtual em Saúde (BVS) e na Scientific Electronic Library Online (SciELO). Para a busca, foram utilizados descritores na língua portuguesa, sendo estes: “Cuidados Paliativos, Enfermagem e Pediatria”, indexadas nos Descritores em Ciências da Saúde (DeCs), foi utilizado booleano AND. Os critérios de inclusão definidos para a seleção dos artigos foram: publicados nos idiomas português, inglês e espanhol; disponíveis gratuitamente e online na íntegra nas bases de dados descritas acima, publicados durante o período de 2015 a 2020. Já, os critérios de exclusão foram estudos como teses, dissertações, trabalhos de conclusão de curso, resumos, anais, editoriais e capítulos de livro. A Figura 2 apresenta o fluxograma da busca de artigos nas respectivas bases de dados.

Figura 2 - Fluxograma do número de artigos encontrados nas respectivas bases de dados.


Fonte: Autores, (2020).

\section{Resultados}

Inicialmente foram encontrados 30 artigos, e após a aplicação dos filtros de seleção, foram incluídos seis. Para a análise dos dados, foi elaborado um quadro analítico com as informações extraídas dos estudos, incluindo o ano de publicação, a base de dados, o periódico, o título do artigo, a autoria e os principais resultados da revisão integrativa. 
Quadro 1: Caracterização dos Estudos Incluídos na Revisão.

\begin{tabular}{|c|c|c|c|c|}
\hline $\begin{array}{c}\text { Ano de } \\
\text { publicação }\end{array}$ & $\begin{array}{c}\text { Base de } \\
\text { Dados }\end{array}$ & Periódico & Título do artigo e Autores & Principais resultados \\
\hline 2015 & LILACS & $\begin{array}{l}\text { Revista } \\
\text { Gaúcha de } \\
\text { Enfermage } \\
\text { m }\end{array}$ & $\begin{array}{l}\text { Palliative care in paediatric } \\
\text { oncology: perceptions, expertise and } \\
\text { practices from the perspective of the } \\
\text { multidisciplinary team. } \\
\text { Silva et al. }\end{array}$ & $\begin{array}{l}\text { O estudo teve como objetivo, conhecer } \\
\text { as percepções, saberes e práticas da } \\
\text { equipe multiprofissional na atenção às } \\
\text { crianças em cuidados paliativos em } \\
\text { unidade de oncologia pediátrica. O } \\
\text { estudo mostra que a equipe sofre, } \\
\text { igualmente, com a morte da criança e, de } \\
\text { forma semelhante à família, move-se em } \\
\text { direção à construção de mecanismos de } \\
\text { enfrentamento para a elaboração do luto. }\end{array}$ \\
\hline 2017 & SciELO & $\begin{array}{ll}\text { Persona } & \text { y } \\
\text { Bioética } & \end{array}$ & $\begin{array}{l}\text { Encontrar sentido para continuar } \\
\text { viviendo el reto al perder un hijo por } \\
\text { cáncer infantil: revisión integrativa. } \\
\text { Moreno, Diaz \& López. }\end{array}$ & $\begin{array}{l}\text { Os autores deste estudo identificaram os } \\
\text { principais aspectos da experiência de } \\
\text { perder um filho em decorrência do } \\
\text { câncer infantil. Em relação a isso, pode- } \\
\text { se ver que esse é um padrão importante } \\
\text { para o desenvolvimento da área de } \\
\text { cuidados paliativos no final da vida e } \\
\text { posteriormente. }\end{array}$ \\
\hline 2018 & BDENF & $\begin{array}{l}\text { Revista de } \\
\text { Enfermage } \\
\mathrm{m} \quad \text { UFPE } \\
\text { Online }\end{array}$ & $\begin{array}{l}\text { Cuidados paliativos pediátricos: } \\
\text { análise de estudos de enfermagem. } \\
\text { Souza et al. }\end{array}$ & $\begin{array}{l}\text { O objetivo deste estudo foi analisar as } \\
\text { evidências científicas acerca dos } \\
\text { cuidados paliativos pediátricos. Este } \\
\text { estudo, mostrou uma literatura focada na } \\
\text { oncologia e assistência ao cuidador, } \\
\text { revelando ainda baixa publicação de } \\
\text { outras condições crônicas na pediatria. }\end{array}$ \\
\hline 2019 & BDENF & $\begin{array}{l}\text { Revista de } \\
\text { Enfermage } \\
\mathrm{m} \quad \text { UFPE } \\
\text { Online }\end{array}$ & $\begin{array}{l}\text { Profissionais de enfermagem: } \\
\text { compreensão sobre cuidados } \\
\text { paliativos pediátricos. } \\
\text { Verri et al. }\end{array}$ & $\begin{array}{l}\text { Os autores trazem como objetivo } \\
\text { investigar a compreensão e a prática dos } \\
\text { profissionais de enfermagem sobre os } \\
\text { cuidados paliativos pediátricos. Sendo } \\
\text { assim, o estudo salienta a necessidade da } \\
\text { inclusão de cuidados paliativos na } \\
\text { formação acadêmica dos profissionais, } \\
\text { favorecendo o conhecimento do tema e } \\
\text { preparando o profissional para lidar com } \\
\text { a morte e o morrer, assim como a } \\
\text { necessidade de um àde que } \\
\text { nas instituições de } \\
\text { proporcione acolhimento frente } \\
\text { dificuldades dos profissionais que atuam } \\
\text { nesse contexto. }\end{array}$ \\
\hline
\end{tabular}




\begin{tabular}{|c|c|c|c|c|}
\hline 2019 & SciELO & $\begin{array}{l}\text { Physis: } \\
\text { Revista de } \\
\text { Saúde } \\
\text { Coletiva }\end{array}$ & $\begin{array}{l}\text { No fio da navalha: a dimensão } \\
\text { intersubjetiva do cuidado aos bebês } \\
\text { com condições crônicas complexas. } \\
\text { Azevedo \& Pfeil. }\end{array}$ & $\begin{array}{l}\text { Os autores trazem a importância de } \\
\text { explorar os desafios advindos do } \\
\text { cuidado de bebês cronicamente } \\
\text { adoecidos e submetidos à dependência } \\
\text { tecnológica na perspectiva dos } \\
\text { profissionais de saúde, vistos como } \\
\text { sujeitos capazes de elaboração psíquica } \\
\text { e transformação de suas práticas } \\
\text { profissionais. O estudo buscou } \\
\text { compreender em profundidade a } \\
\text { realidade e a singularidade da } \\
\text { experiência cotidiana, sendo central a } \\
\text { valorização da elaboração psíquica e da } \\
\text { produção de sentidos dos sujeitos. }\end{array}$ \\
\hline 2020 & LILACS & $\begin{array}{l}\text { Revista de } \\
\text { Pesquisa: } \\
\text { Cuidado é } \\
\text { Fundamenta } \\
\text { 1 Online }\end{array}$ & $\begin{array}{l}\text { Doença crônica e cuidados } \\
\text { paliativos pediátricos: saberes e } \\
\text { práticas de enfermeiros à luz do } \\
\text { cuidado humano. } \\
\text { Buck et al. }\end{array}$ & $\begin{array}{l}\text { O estudo teve como objetivo, analisar } \\
\text { saberes práticas } \\
\text { de enfermeiros assistenciais } \\
\text { sobre cuidados } \\
\text { paliativos à criança com doença crônica, } \\
\text { à luz da Teoria do Cuidado Humano. } \\
\text { Este mostrou que existem fragilidades } \\
\text { no saber das participantes que } \\
\text { dificultaram a definição de cuidados } \\
\text { paliativos, medidas de conforto, } \\
\text { comunicação e cuidado espiritual } \\
\text { também foram abordados como meios } \\
\text { para amenizar o sofrimento de crianças } \\
\text { com as doenças. }\end{array}$ \\
\hline
\end{tabular}

Fonte: Fonte: Autores, (2020).

Através da análise, foi possível observar a maior frequência de estudos realizados nas regiões norte, sul, sudeste, sendo que a região norte, apresenta um artigo no estado do Pará (PA) no ano de 2018, na região sul um artigo no estado do Rio Grande do Sul, no ano de 2015, na região sudeste apresentam-se dois artigos no estado do Rio de Janeiro (RJ) no ano de 2019 e 2020 , um no estado de São Paulo (SP) no ano de 2019. Além do Brasil, outro país da América do Sul teve um artigo publicado na Colômbia (CO) no ano de 2017.

Mediante análise dos artigos, foi evidenciado que os cuidados paliativos em pediatria, são adotados, na maior parte das situações, em pacientes com doenças oncológicas (Souza et al., 2018). Sendo que no estudo de Moreno, Diaz \& Lopez, 2017, os autores identificaram os principais aspectos vividos pelas famílias que tiveram a perda de um filho com câncer, e as experiências sob os cuidados prestados no final da vida e os desafios enfrentados para dar continuidade à vida após a perda.

Silva et al, 2015, teve como propósito do estudo conhecer as experiências e práticas que a equipe vivencia durante a internação da criança em cuidados paliativos (Silva et al., 2015). Assim como o estudo de Buck et al., 2020 e de Verri et al., 2019 que buscaram investigar e compreender a forma em que a equipe de enfermagem se relaciona e presta assistência para a criança, ressaltando a importância de ter conhecimento prévio na área para ter domínio da execução das atividades propostas. Desta forma, 
é possível criar um vínculo com a criança a fim de facilitar na construção de um plano que auxilie ainda mais no processo terapêutico.

\section{Discussão}

O método de análise da revisão integrativa baseou-se na categorização das informações coletadas. Assim, os dados foram interpretados e agrupados em duas categorias para compreensão do fenômeno: abordagens adotadas pela enfermagem em pacientes pediátricos em cuidados paliativos e estratégias de enfrentamento da equipe diante das situações vivenciadas no processo de cuidados paliativos.

\subsection{Abordagens adotadas pela enfermagem em pacientes pediátricos em cuidados paliativos}

Souza et al., 2018, busca analisar evidências científicas em cuidados paliativos, focando somente na oncologia, enquanto Buck et al., 2020, analisa a assistência em cuidados paliativos em crianças com doenças crônicas e oncológicas, que são condições que apresentam um curso progressivo, com prognósticos incertos. Estas limitam a vida do portador, expondo-o a períodos de remissão e exacerbação clínica, gerando a necessidade de cuidados contínuos e por vezes complexos, seja a nível domiciliar, ambulatorial ou hospitalar, nesse meio existe um espaço para o cuidado paliativo, e quando o foco se dá nas crianças, como ser humano em desenvolvimento, visa se proporcionar ainda mais qualidade de vida.

Para Silva et al., 2015, o conhecimento dos profissionais dessa área para atuar nas intervenções é fundamental, assim como Verri et al., 2019, que salienta sobre a necessidade de incluir conteúdos em cuidados paliativos ainda na formação acadêmica, a fim de preparar o profissional para lidar e atuar nesse contexto. A equipe não atua somente no cuidado, mas sim como uma rede de apoio a todos que passam por essa experiência. Na opinião de Azevedo \& Pfeil, 2019, a enfermagem precisa desenvolver técnicas que atinjam as necessidades de assistência, particularizando o cuidado de cada caso e evitando que eles se sintam incapacitados por trabalhar com essa situação. Por isso, proporcionar conforto e melhor qualidade de vida, é o primeiro meio a ser oferecido pela equipe durante esse processo, diz Silva et al., 2015. Respeitar as vontades e necessidades do paciente com estratégias de forma multiprofissional que focam na individualidade sem subestimar a competência dos pais e familiares, é uma forma de não os deixar desamparados quando necessitam de auxílio nos momentos de fraqueza e solidão (Silva et al., 2015).

Para aprimorar estes cuidados, uma equipe multiprofissional deve unir suas habilidades a fim de ajudar o paciente a adaptar-se às mudanças de vida impostas pela doença e proporcionar momentos de interação entre o paciente e sua família, que faz com que ele não tenha o pensamento só na doença e vivencie um dia após o outro. Por isso, a enfermagem é fundamental para a equipe de cuidados paliativos pela essência de sua formação que se baseia na arte do cuidar e por buscar realizar ações de conforto, além dos cuidados básicos que o paciente necessita, sempre visando realizar os desejos, vontades e anseios do paciente (Verri et al., 2019).

Prestar assistência em cuidados paliativos, é poder propiciar para aquela pessoa e família, o que está necessitando, independentemente da perspectiva de um falecimento. Moreno, Diaz \& López, 2017 dizem que nesses momentos, a equipe pode conceder exceções a regras que antes eram rígidas com o intuito de trazer carinho e conforto as crianças . Existem protocolos, materiais e procedimentos que impactam positivamente a criança. A equipe deve compreender essas peculiaridades tornando assim um processo mais humano.

Na construção coletiva do plano de cuidado, deve-se atentar a comunicação, o respeito e o relacionamento interpessoal, como a essência do cuidado para esses momentos mais difíceis, ou seja, a enfermagem visa a arte e a ciência de prestar assistência ao doente nas suas necessidades básicas e, em se tratando de cuidados paliativos, contribui para uma sobrevida mais digna e uma morte mais tranquila (Silva et al., 2015). Buck et al., 2020, observam que a equipe procura prestar uma assistência humanizada, sempre com o intuito de fazer o possível para que o paciente não sinta dor, uma maneira de promover o alívio da dor não só de 
forma medicamentosa, é garantir amparo e segurança ao indivíduo e seu familiar, afinal, oferecer um sistema de suporte para que o paciente possa ser ativo de acordo com as suas possibilidades, faz com que o tratamento se torne mais eficaz, possibilitando uma melhor aceitação.

Entende-se que para haver um bom atendimento, a equipe deve andar em harmonia para que assim, possa se compreender a importância de um atendimento eficaz diante dessas situações, por isso o envolvimento da equipe, tão quanto a empatia fazem com que sejam ofertados cuidados em que é possível transcender o cuidado físico e ir ao encontro do mundo subjetivo, emocional e espiritual do ser cuidado e do enfermeiro, vivenciando momentos terapêuticos repletos de amor, carinho, compaixão, empatia e acolhimento (Buck et al., 2020).

\subsection{Estratégias de enfrentamento da equipe diante das situações vivenciadas no processo de cuidados paliativos}

Em relação ao desenvolvimento do enfrentamento psicológico da equipe, Verri et al., 2019, figuraram como principal fonte de debilidade, a falta de preparo em cuidados paliativos ainda no meio acadêmico, o que faz com que diante dessas situações alguns profissionais apresentem dificuldades relacionadas à compreensão sob os cuidados paliativos e resistência em atuar com pacientes pediátricos que estão sob esse cuidado, destacando-se os sentimentos de fracasso e de tristeza ao lidarem com a situação (Verri et al., 2019).

Com o avanço da medicina, o óbito passou a ocorrer a nível hospitalar, deixando de ocorrer na casa do doente, o qual era muito comum, uma vez a nível hospitalar, a equipe antes dedicada ao máximo à cura da enfermidade, depara-se com a terminalidade e com a dor da família, o que exige dos profissionais, além de conhecimento técnico-científico, também preparo emocional para auxiliar as famílias (Silva et al., 2015). Por isso, Verri et al., 2019 colocam o quão necessário é introduzir vivências nessa área a fim de preparar e favorecer o profissional para que diante dessas necessidades sinta-se seguro a criar um vínculo com a família e paciente, tornando a situação mais agradável pela forma que é conduzida. Frente a essa temática, Silva et al., 2015, mencionam a necessidade do trabalho da equipe multiprofissional para o sucesso no cuidado à criança com doenças crônicas e oncológica, possibilitando controle na forma de prestar assistência e propiciar mais tranquilidade e segurança para todos que se envolvem nesse meio.

Uma forma de interação que facilita o trabalho entre a equipe e o paciente, é entender que para se ter eficácia naquilo que é feito, é necessário deixar de lado as emoções e ir em busca dos objetivos traçados pela equipe, independentemente deste ser um momento delicado (Verri et al., 2019). Dentre os profissionais de saúde, Buck et al.,2020, dizem que os enfermeiros são os que possuem o maior potencial para se conectar com a criança em sofrimento e, assim, desenvolver uma relação de proximidade empática e harmônica, capaz de transcender o cuidar para as dimensões emocionais, sociais e espirituais e, portanto, prover um cuidado humano em sua assistência com a pediatria (Buck et al.,2020).

Uma grande dificuldade vista pelos profissionais da saúde, é voltada ao enfrentamento psicológico por conta das situações que eles vivenciam no seu dia a dia de trabalho. Machado, Oliva \& Bengozi, 2019, dizem que a enfermagem é uma das categorias com maior desgaste emocional, devido à interação com os pacientes, as constantes internações, muitas vezes acompanhando o sofrimento, como a dor, a doença e a morte do ser cuidado. Por isso Verri et al., 2019, relatam que a criação de um grupo de debates também é um meio importante para todos que participam desse processo, pois com essa tarefa pode-se aprimorar as estratégias de enfrentamento do processo de tratamento por parte do familiar e da equipe, com intuito de ser de apoio, aberto e acolhedor, tendo assim a oportunidade para que esses encontrassem um espaço para falar livremente de suas tensões e dificuldades enfrentadas no cotidiano hospitalar, de compartilhar suas emoções com pessoas que vivenciam a mesma situação e de se sentirem escutados, compreendidos e amparados por profissionais que não focam somente no paciente, mas também na família e na própria equipe que acompanha todo processo (Verri et al., 2019).

Conforme a expressão trazida por Azevedo \& Pfeil, 2019, no “fio da navalha” remete às incertezas, limites e desafios 
enfrentados pelos profissionais de saúde no cuidado ao bebê gravemente enfermo, no que se refere a lidar com o investimento na vida, mas também com o risco de morte. O contato com a fragilidade humana e as vivências perante a precariedade e finitude podem favorecer a aceitação dos próprios limites e ressignificações quanto aos sentidos não só do cuidado em saúde, mas talvez da própria vida, evidenciando a natureza do trabalho psíquico que se desenvolve neste cenário (Azevedo e Pfeil, 2019). Preparar o profissional para lidar com a morte e o morrer para Borges \& Mendes, 2012, assim como a necessidade de um espaço nas instituições de saúde que proporcione acolhimento frente às dificuldades dos profissionais que atuam nesse contexto, é uma forma de ajudar o profissional a desenvolver melhores suas atividades, pois essas vivências fazem com que ele por vezes, sinta vontade de desistir, por ver todo sofrimento e angústia do paciente e suas famílias, dessa maneira é possível encorajá-lo, através do reconhecimento sob ele, a fim de que mesmo sendo um momento de tristeza, ele sinta se motivado a dar o seu melhor (Verri et al., 2019).

Frente a esses momentos de tamanha dor, a equipe foca não somente no cuidado, mas sim em se entregar ao paciente e seu familiar, buscando propiciar tudo que ela está necessitando, independente de não haver a cura. É um momento em que se propõe o cuidado em relação à dor, a estar confortável, manter as pessoas que gostam próximas, interagir de formas que venham a somar para aquele ser indefeso. Nesse ciclo, é possível refletir sobre a importância do profissional nesse momento delicado da vida de uma família e compreender que o principal objetivo é resgatar a humanização. O profissional que trabalha no setor oncológico pediátrico se depara com mudanças significativas, no que se refere a assistência, onde é formado para trabalhar com promoção de saúde e passa a lidar com morte rotineiramente (Fernandes et al., 2013).

A tão esperada cura começa a dar lugar a qualidade de vida e assim, a mesma equipe que sofre as ambivalências de suas práticas, só tem poder de garantir à criança a dignidade da vida, até o momento de sua morte. Moreno, Diaz \& López, 2017, relatam que a atuação em ambientes que exijam a compreensão do cuidador, torna aquela situação mais devastadora, facilitando a criação de um vínculo entre o profissional, paciente e familiar, e isso faz com que haja maior facilidade de se relacionar e manter um cuidado ativo. Este envolvimento passa ser mais afetivo e emocionante, pois é possível perceber a condição entre o sentir e o agir, pois o vínculo que se cria é o mesmo que faz com que os profissionais sofram quando o óbito da criança se aproxima, e são essas as situações em que os profissionais precisam se fazer fortes para encarar sua jornada com um novo paciente, pois os sentimentos são mútuos, e eles sabem que diante dessas condições estes não são expressados pois ali , o profissional serve como suporte para as famílias expressar suas emoções, e não um momento de eles demonstrarem suas dificuldades e desequilíbrios em encarar essas situações (Moreno, Diaz \& López, 2017).

Frente a momentos de sofrimento e exigências, Azevedo \& Pfeil, 2019, contam que os profissionais são pouco resolutivos a assumirem a tradicional postura de não se envolver, pois trabalhar com crianças vai além do envolvimento emocional, são períodos que mexem com o psicológico e exigem conhecimento, empatia e humanização, o que possibilita criar laços com a família a fim de facilitar as diferentes formas de cuidados que podem ser oferecidas a essas crianças. Sendo assim, Silva et al., 2015, dizem que a equipe busca inserir a família na construção do projeto terapêutico singular para nortear esse momento do tratamento oncológico e permitir que eles vivenciem todas as etapas da doença, pois é uma forma de eles se inserir no cuidado, e fazer com que eles possam compartilhar essas experiências que proporcionaram qualidade ao seu ente querido.

\section{Conclusão}

Levando em consideração o exposto, a partir da revisão dos artigos, denota-se uma fundamental importância da equipe de enfermagem no processo de cuidados paliativos em pediatria, uma vez que a equipe quanto mais próxima do paciente, possibilita um melhor relacionamento com a criança, podendo então criar um vínculo e através disso prestar um cuidado digno e humanizado. Para tanto a enfermagem precisa de um apoio psicológico e para que consiga atuar de maneira humana e eficiente nesse cenário complexo que envolve famílias que requerem amparo e apoio. Portanto, além do conhecimento técnico científico, 
dedicação e compreensão sobre aquilo que deve ser feito, é a melhor maneira de prestar assistência de forma humanizada, oferecendo comodidade e qualidade de vida.

Ter conhecimento prévio sobre a área é uma forma de estar preparado para suportar os diferentes casos que são vistos no dia a dia de uma equipe. Em razão disso, trabalhar as emoções e a forma de se relacionar com o paciente e seu familiar perante esse cenário, faz com que haja um melhor entendimento sobre a temática, bem como, torna mais transparente a condução e acessibilidade aos que vivenciam tais eventos.

Uma vez que esse é um ato que visa melhorar a qualidade de vida do paciente e consequentemente da sua família, sugerimos que continuem sendo realizados novos estudos acerca desse tema, a fim de melhorar ainda mais o modo de conduzir e abordar as famílias que vivenciam este processo. Além disso, vale ressaltar que tem-se notado um aumento significativo no desenvolvimento de doenças crônicas nas crianças, exigindo assim, ainda mais preparo dos profissionais para explanar as dúvidas dos familiares, deixando-os mais seguros e confortáveis diante das situações. Sendo assim, é de suma importância que os profissionais tenham sua saúde mental muito bem desenvolvida, para que não acabem se desgastando e gerando mais um processo de doença.

\section{Referências}

Alves, R. F., et al. (2015). Cuidados paliativos: desafios para cuidadores e profissionais de saúde:Revista de Psicologia, v. 27, n. 2, p. 165-176.

Azevedo, C. S. \& Pfeil, N. V. (2019). No fio da navalha: a dimensão intersubjetiva do cuidado aos bebês com condições crônicas complexas: Revista de Saúde Coletiva, v. 29(4), e290406.

Borges, M. S., \& Mendes, N. (2012). Representações de profissionais de saúde sobre a morte e o morrer. Rev Bras Enferm.; 65(2): 324-31.

Buck, E. C. S., et al. (2020). Doença crônica e cuidados paliativos pediátricos: saberes e práticas de enfermeiros à luz do cuidado humano. Rev. Pesqui. Univ. Fed. Estado Rio Janeiro. 12: 682-688.

Carvalho, M. T. (2009). Manual de Cuidados Paliativos. Atlas.

Carvalho, M. (2016). Incidência de câncer infantil. http://www.aacc.org.br/incidencia-de-cancer-infantil.

Cavalcante, A. K. C. B., et al. (2015). Cuidado seguro ao paciente: contribuições da enfermagem. Rev. Cubana de Enfermaría. v 31 , n 4.

Fernandes, M. A. et al. (2013). Percepção dos enfermeiros sobre o significado dos cuidados paliativos em pacientes com câncer terminal. Ciência \& Saúde Coletiva. 18(9):2589-2596.

Hermes, H. R. \& Lamarca, I. C. A. (2013). Cuidados paliativos: uma abordagem a partir das categorias profissionais de saúde. Ciência \& Saúde Coletiva, 18(9):2577-2588.

Iglesias, S. B., Zoller, A. C. R. \& Constantino, C. F. (2016). Cuidados Paliativos Pediátricos. Residência Pediátrica. 6(supl 1):46-54.

Inca, M.S. (2018). Cuidados Paliativos. https://www.inca.gov.br/tratamento/cuidados-paliativos.

Machado, J. A. M., Oliva, L. L. \& Bengozi, T. M. (2019) Dificuldades da equipe de enfermagem frente aos cuidados paliativos em pediatria: uma revisão integrativa. Rev. Terra \& Cult.: v. 35, n. especial.

Melo, A. C., Valero, F. F. \& Menezes, M. (2013). A intervenção psicológica em cuidados paliativos. Psicologia, saúde e doença. 14(3), 452-469.

Mendes, K. D. S., Silveira, R. C. C. P. \& Galvão, C. M. (2008). Revisão integrativa: método de pesquisa para a incorporação de evidências na saúde e na enfermagem. Rev. Texto Contexto Enferm.v. 17, n. 4, p. 758-764.

Misko, M. D., et al. (2015). A experiência da família da criança e/ou adolescente em cuidados paliativos: flutuando entre a esperança e a desesperança em um mundo transformado pelas perdas. Rev. Latino-Am. Enfermagem. 23(3):560-7.

Moreno, S. C., Díaz, L. C.\& López, R. R. (2017). Encontrar sentido para continuar viviendo el reto al perder un hijo por cáncer infantil: revisión integrativa. Pers. bioet ; 21(1): 46-61.

Silva, A. F., et al. (2015). Cuidados paliativos em oncología pediátrica: percepciones, saberes y prácticas bajo la perspectiva de un equipo multidisciplinario. Rev Gaúcha Enferm.36(2):56-62.

Souza, M. T., Silva M. D. \& Carvalho, R. (2010). Revisão integrativa: o que é e como fazer. Journal Einstein. 8(1 Pt 1):102-106.

Souza, T. C. F., et al. (2018). Cuidados paliativos pediátricos: análise de estudos de enfermagem. Rev. enferm. UFPE on line ; 12(5): 1409-1421. 
Research, Society and Development, v. 10, n. 5, e13910514848, 2021

(CC BY 4.0) | ISSN 2525-3409 | DOI: http://dx.doi.org/10.33448/rsd-v10i5.14848

Verri, E. R., et al. (2019). Profissionais de enfermagem: compreensão sobre cuidados paliativos pediátricos. Rev. enferm. UFPE. 13(1): 126-136. 